\title{
Statistical Unigram Analysis for Source Code Repository
}

\author{
Weifeng Xu \\ Department of Computer Science \\ Bowie State University \\ Bowie, MD, USA \\ wxu@bowiestate.edu
}

\author{
Dianxiang $\mathrm{Xu}$ \\ Department of Computer Science \\ Boise State University \\ Boise, ID, USA \\ dianxiangxu@boisestate.edu
}

\author{
Omar El Ariss \\ Department of Computer Science \\ The Pennsylvania State University- \\ Harrisburg \\ Middletown, PA, USA \\ oue1@psu.edu
}

\author{
Yunkai Liu \\ Department of Computer \& \\ Information Science \\ Gannon University \\ Erie, PA, USA \\ liu006@gannon.edu
}

\author{
Abdulrahman Alatawi \\ Department of Computer Science \\ Bowie State University \\ Bowie, MD, USA \\ Alatawi1116@students.bowiestate.e \\ $\mathrm{du}$
}

\begin{abstract}
Unigram is a fundamental element of n-gram in natural language processing. However, unigrams collected from a natural language corpus are unsuitable for solving problems in the domain of computer programming languages. In this paper, we analyze the properties of unigrams collected from an ultralarge source code repository. Specifically, we have collected 1.01 billion unigrams from 0.7 million open source projects hosted at GitHub.com. By analyzing these unigrams, we have discovered statistical patterns regarding (1) how developers name variables, methods, and classes, and (2) how developers choose abbreviations. Our study describes a probabilistic model for solving a well-known problem in source code analysis: how to expand a given abbreviation to its original indented word. It shows that the unigrams collected from source code repositories are essential resources to solving the domain specific problems.
\end{abstract}

Keywords-programming language; source code; n-gram; unigram; abbreviations; ultra-large-scale analysis

\section{INTRODUCTION}

Natural languages and computer programming languages are both used to communicate and solve real-world problems. In the domain of software engineering, solutions to real-world problems specified in requirements documentation are often expressed in a natural language. However, natural languages cannot be used for implementing the software solutions due to the ambiguity, either semantically or syntactically [1] [2]. The implementation of such solutions uses the source code, which can be written in various computer programming languages. Programming languages are formally constructed languages designed to communicate with a specific machine. For example, the following Python and Java source code describe a solution to printing guests' names from a list of invited people.

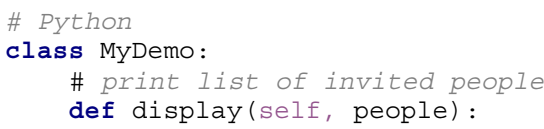

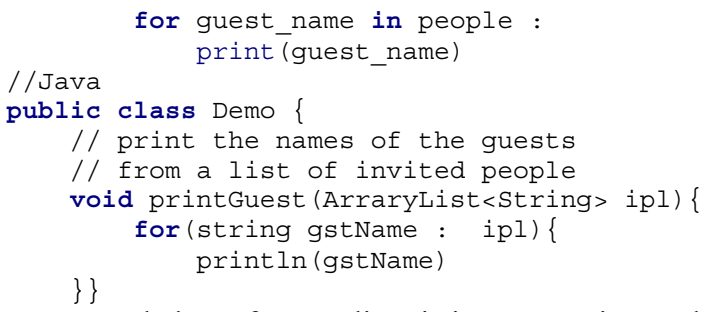

Nevertheless, from a linguistic perspective and based on Chomsky's hierarchy for languages [3], programming languages are similar to natural languages since most of the grammatical features of both types of languages can be represented using a context-free grammar. In addition, developers use the vocabularies of a natural language, i.e., English words, including print, guest, invited, and name, to name identifiers, such as variables, methods, and classes. The terms variable, method, and class are formally defined constructs in objected-oriented languages. Reusing English words in source code ensures the readability of source code because (1) structured programming [4] requires meaningful names for identifiers (2) English words are meaningful for communication in the real-world (3) English names persevere the same or similar meanings in both realworld and the domain of software engineering. In the previous sample code, developers use the English words guest and display to name a variable and a method for displaying information.

In practice, naming program constructs is more complicated than simply picking up English words: (1) The names of the constructs are often mixtures of a single English word, multiple English words, and abbreviations. For example, the variable name gst Name consists of one abbreviation gst and one English word name to represent the name of a guest. (2) The same English word used in the domain of natural language may represent different meaning in programming languages, for example, the word class. The difference impacts the understandability of source code. Regardless of the 
syntactical and semantical complexity of the constructs' names, its elements mainly consist of one or more unigrams. Each unigram is either an English word or an English abbreviation. Therefore, we are interested in investigating the properties of unigrams in computer programs, aiming to understand: (1) how developers name variables, methods and classes and (2) how developers choose abbreviations.

We conduct our study using the variable, method and class names extracted from the entire source code repository hosted on GitHub.com [5] during the year of 2015. The GitHub repository contains 699,331 open source projects [6]. Each project can be written in different programming languages. The contributions of the paper are as follows:

(1) This is the first attempt to analyze the properties of unigrams in computer programs at such an ultra-large scale.

(2) The unigrams collected from the source code repository in this study can solve such domain specific problem, as expanding name abbreviations using unigram models.

(3) The entire corpus, including unigrams, abbreviations, and results, are available online. It provides a useful benchmark for future research.

The rest of this paper is organized as follows: Section II reviews the related work. Section III describes the process of unigram collection from GitHub. Section IV analyzes the properties of unigrams. Section V presents the empirical study. Section VI concludes this paper.

\section{RELATED WORK}

An n-gram is a contiguous sequence of $n$ items from a given sequence of text. N-grams, particularly unigrams and bigrams, collected from texts are extensively used in text mining and natural language processing, including machine translation, speech recognition, spelling correction, etc. Linguistics Data Consortium has published the n-gram data in 2006, including 16 million of unigrams and 315 bigrams collected from one Terabyte web collection [7]. The Google Ngram Viewer [8] is an online tool that charts frequencies of given unigrams or bigrams found in printed sources between the years 1500 and 2008 .

Although n-gram analysis has been introduced in other domains, n-gram analysis has not been well-studied in the context of programming languages and specifically for the analysis of the naming conventions of identifiers. Therefore, there is very little research done that is closely related to our proposed work. Allamanis and Sutton [9] use a trigram language model based on a corpus of 14,807 Java programs to come up with various metrics including code complexity and variable originality. On the other hand, the authors use n-grams on single projects to suggest proper coding conventions such as formatting and identifier naming [10]. Allamanis et al. [11] use a neural logbilinear model to improve existing code by suggesting names for methods and classes. Finally, Raychev et al. [12] introduce a statistical model based on conditional random fields (CRFs) to predict the types of variable names and the names of variables in obfuscated JavaScript code.

\section{UNIGRAM COLLECTION}

Variables, methods, and classes are essential to construct types of source code. The names of these constructs are categorized into two groups shown below:

- Unigram name: a name of construct that consists of a single unigram, which is either an English word or an abbreviation of an English word. They are referred to as unigram English names and unigram abbreviation names, respectively. For example, a single English word display can be used for naming a method; the abbreviation demo is used to name a class (see Table 1). Thus, the word display is a unigram English name and demo is a unigram abbreviation name.

- Multigram name: a name of a construct that consists of multiple unigrams. For example, gstName is a multigram name, which consists of two unigrams gst and name. The unigram gst is the abbreviation of the English word guest. Other examples are shown in Table 1 .

Table 1. Categories and examples of variable, method, and class names

\begin{tabular}{|c|c|c|c|}
\hline & Variable & Method & Class \\
\hline Unigram name & guest, people, gst & display & demo \\
\hline Multigram name & $\begin{array}{c}\text { guest_name, gstName, } \\
\text { ipl }\end{array}$ & printGuest & myDems \\
\hline
\end{tabular}

We use unigrams collected from source code repository, i.e., GitHub, to analyze patterns of unigram names and multigram names. Figure 1 shows the process of unigram collection and analysis.

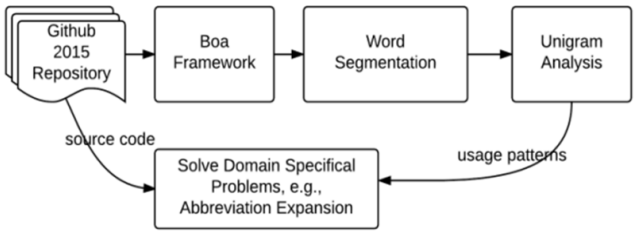

Figure 1. Block diagram of unigram collection and analysis

The process mainly consists of three components:

- Boa Framework [13]. It is a language and infrastructure for extracting syntactic information from source code in GitHub, including variable, method, and class names. The framework converts source code to AST trees, and then a visitor traverses the trees to collect names.

- Word segmentation [14]. This phase determines where the word boundaries are for a given multigram name. Processing natural language, such as English, doesn't normally need to perform word segmentation because words in English sentences are most of the time separated by white spaces. However, a construct's name often is a multigram name without spaces. For example variable names such as username and user_name require segmentation. 
- Unigram analysis. This phase utilizes Apache Hadoop framework [15] to study the properties of unigrams collected from source code. The properties will be used to solve domain specific problems, e.g., expanding abbreviations used in source code, which will be covered in section $\mathrm{V}$.

Table 2 shows: (1) 0.70 billion constructs are extracted from nearly 0.7 million projects. (2) Over one billion unigrams are collected from the constructs.

Table 2. The total number of constructs, unigrams extracted from GitHub (in millions)

\begin{tabular}{|c|c|c|c|c|}
\hline & Variable & Method & Class & Total \\
\hline The number of constructs & 419 & 161 & 21 & 701 \\
\hline $\begin{array}{c}\text { The number of unigrams extracted } \\
\text { from constructs }\end{array}$ & 636 & 396 & 57 & 1069 \\
\hline
\end{tabular}

\section{ANALYSIS OF UNIGRAMS}

Unigrams collected from the GitHub source code repository are different from unigrams collected from natural language corpus, i.e., news archives. In this section, we compute the most commonly used English words and abbreviations in the source code, and then we reveal two important statistical properties of abbreviations.

\section{A. Most Commonly Used English Words in Source Code}

We have extracted the most commonly used English words from identifier names: variable, method, and class names, respectively. Table 3 shows the top 70 most frequently used English words for naming variables from 636 million unigrams extracted from variable names.

Table 3. The top 60 most frequently used English words for naming

\begin{tabular}{|c|c|c|c|c|c|c|c|}
\hline \multicolumn{8}{|c|}{ variables } \\
\hline Word & $\begin{array}{l}\text { Freq } \\
.(\mathbf{k}) \\
\end{array}$ & Word & $\begin{array}{l}\text { Freq } \\
.(\mathbf{k}) \\
\end{array}$ & Word & $\begin{array}{l}\text { Freq } \\
.(\mathbf{k}) \\
\end{array}$ & Word & $\begin{array}{l}\text { Freq } \\
.(\mathbf{k}) \\
\end{array}$ \\
\hline name & 9865 & node & 2306 & class & 1572 & $\begin{array}{c}\text { respons } \\
\mathrm{e}\end{array}$ & 1333 \\
\hline id & 7920 & count & 2301 & parent & 1560 & code & 1320 \\
\hline value & 7354 & item & 2255 & element & 1550 & line & 1306 \\
\hline type & 5847 & field & 2239 & length & 1549 & action & 1292 \\
\hline key & 4235 & $\begin{array}{c}\text { messag } \\
\mathrm{e}\end{array}$ & 2238 & source & 1534 & height & 1286 \\
\hline result & 3983 & to & 2228 & string & 1533 & instance & 1282 \\
\hline data & 3681 & view & 2206 & input & 1515 & current & 1282 \\
\hline index & 3680 & start & 2179 & default & 1510 & $\log$ & 1259 \\
\hline $\begin{array}{c}\text { contex } \\
\mathrm{t}\end{array}$ & 3399 & state & 2078 & target & 1501 & test & 1249 \\
\hline file & 3155 & event & 1941 & $\max$ & 1482 & number & 1227 \\
\hline list & 3115 & time & 1935 & service & 1446 & listener & 1220 \\
\hline in & 3055 & map & 1909 & offset & 1410 & column & 1218 \\
\hline text & 2590 & out & 1864 & end & 1393 & content & 1210 \\
\hline new & 2530 & request & 1758 & width & 1387 & label & 1204 \\
\hline size & 2502 & user & 1643 & date & 1382 & last & 1191 \\
\hline is & 2390 & info & 1640 & tag & 1371 & buffer & 1175 \\
\hline path & 2334 & object & 1613 & $\begin{array}{c}\text { serial } \\
\text { versioni } \\
\mathrm{d}\end{array}$ & 1342 & error & 1009 \\
\hline
\end{tabular}

Table 4 shows that the same English words are ranked differently in GitHub and natural language corpus. For example, the English word name is ranked the first in GitHub and the $108^{\text {th }}$ in the natural language corpus [16], respectively. Similarly, we have computed the top 100 most frequently used English words for naming methods and classes (see Appendix).
Table 4. Different word frequency ranks in source code (SC) and natural

\begin{tabular}{|c|r|r|c|r|r|c|r|r|}
\hline \multirow{2}{*}{ Word } & \multicolumn{1}{|c|}{ Ranking } & \multirow{2}{*}{ Word } & \multicolumn{2}{|c|}{ Ranking } & \multirow{2}{*}{ Word } & \multicolumn{2}{|c|}{ Ranking } \\
\cline { 2 - 3 } & SC & NC & & SC & NC & & SC & NC \\
\hline name & 1 & 108 & node & 18 & 3407 & class & 35 & 388 \\
\hline id & 2 & 654 & count & 19 & 2011 & parent & 36 & 1771 \\
\hline value & 3 & 1146 & item & 20 & 214 & element & 37 & 2222 \\
\hline type & 4 & 253 & field & 21 & 574 & length & 38 & 1155 \\
\hline key & 5 & 569 & message & 22 & 149 & source & 39 & 419 \\
\hline result & 6 & 611 & to & 23 & 4 & string & 40 & 1567 \\
\hline data & 7 & 131 & view & 24 & 79 & input & 41 & 1438 \\
\hline index & 8 & 276 & start & 25 & 474 & default & 42 & 1538 \\
\hline context & 9 & 2022 & state & 26 & 111 & target & 43 & 1584 \\
\hline file & 10 & 281 & event & 27 & 624 & max & 44 & 1428 \\
\hline list & 11 & 107 & time & 28 & 50 & service & 45 & 97 \\
\hline in & 12 & 6 & map & 29 & 197 & offset & 46 & 5179 \\
\hline text & 13 & 349 & out & 30 & 60 & end & 47 & 317 \\
\hline new & 14 & 27 & request & 31 & 627 & width & 48 & 3060 \\
\hline size & 15 & 337 & user & 32 & 185 & date & 49 & 102 \\
\hline is & 16 & 8 & info & 33 & 160 & tag & 50 & 2454 \\
\hline path & 17 & 1790 & object & 34 & 1150 & serial & 51 & - \\
\hline
\end{tabular}

Without surprise, we have observed the following:

(1) Over $95 \%$ of the English words for naming variables and classes are nouns.

(2) $53 \%$ of English words are used for naming both variables and classes.

(3) Among all the top 100 most frequently English words used for naming variables, $42 \%$ of them is used for naming methods, $34 \%$ of them are verbs, and $12 \%$ are prepositions. It is consistent with the purpose of the method construct in object-oriented programming: the manipulation of variables.

\section{B. Patterns of Choosing Abbreviations in Source Code}

In this subsection, we will observe patterns between abbreviations and their original intended English words from small examples, develop an algorithm to extract all possible abbreviation and English word pairs from the ultra-large-scale source code repository. Table 5 shows a small set of applications used to observe abbreviation patterns. They were selected because of their relative popularity, diversity in terms of development maturity and application domain, and availability publicly in open source software repositories.

Table 5. Subject programs used in our experiments to observe patterns

\begin{tabular}{|c|c|c|c|}
\hline Program & Version & KLOC & Description \\
\hline JasperReports & 2.0 .4 & 34.04 & Dynamic content \\
\hline JFreeChart & 1.0 .19 & 57.83 & Data rep. \\
\hline SoapUI & 2.0 .1 & 30.48 & Web service \\
\hline Freecol & 0.7 .3 & 27.21 & Game \\
\hline GanttProject & 2.7 & 28.30 & Scheduling \\
\hline Junit & 4.4 & 0.948 & Software dev. \\
\hline Avuze & 5.5 .0 .0 & 163.53 & Online file share \\
\hline Hibernate & 2.1 .8 & 21.49 & Database \\
\hline JEdit & 4.2 & 32.60 & Text editor \\
\hline DataCraw & 3.4 .5 & 20.20 & Data management \\
\hline Xholon & 0.7 & 23.39 & Simulation \\
\hline Jsch & 0.1 .51 & 7.39 & Security \\
\hline Domination & 1.0 .9 .7 & 8.32 & Game \\
\hline JMencode & 0.64 & 1.33 & Video encoding \\
\hline
\end{tabular}


We review the source code and have observed the following patterns (shown in Table 6) between abbreviations and their original intended English words:

- Pattern one: There are mainly two types of strategies for choosing abbreviations. (a) Consecutive Characters Strategy, which uses the first $n$ consecutive characters as the abbreviation for a given English word and (b) Nonconsecutive Characters Strategy, which uses $n$ nonconsecutive characters as the abbreviation. The two different strategies produce two different abbreviation types: Consecutive Characters Abbreviation (CCA) and Nonconsecutive Characters Abbreviation (NCA).

- Pattern two: The first letter matters. Regardless of the different type of abbreviation choosing strategies, the first letter of the abbreviation is always the first letter of its original intended English word.

- Pattern three: The order of the characters in abbreviations and its intended English word is consistent. Regardless of the different types of abbreviations, developers choose the characters from left to right from the intended English word as its abbreviation. It is evident for CCA because it uses $n$-consecutive characters from the original intended English word as its abbreviation. For example, using str as the abbreviation for the word string. For NCA, the abbreviation src is picked up from the word source at positions 0,3 , and 4 .

- Pattern four: The majority of abbreviations use less than four characters to represent unigram variable names. We will verify the pattern in section $\mathrm{C}$ once we extract all abbreviations and their intended words.

Table 6. Observed patterns between abbreviations and their original intended English words

\begin{tabular}{|c|c|c|c|c|}
\hline \multirow[t]{2}{*}{ Abbreviation Type } & \multicolumn{2}{|c|}{$n=1$} & \multicolumn{2}{|c|}{$n=2$} \\
\hline & Name & Abbr. & Name & Abbr. \\
\hline \multirow{4}{*}{$\begin{array}{l}\text { n-Consecutive Characters } \\
\text { as Abbreviation (CCA) }\end{array}$} & node & $\mathrm{n}$ & exception & ex \\
\hline & value & $\mathrm{V}$ & event & ev \\
\hline & list & 1 & iterator & it \\
\hline & handler & $\mathrm{h}$ & extent & ex \\
\hline \multirow{4}{*}{$\begin{array}{c}\text { n-Nonconsecutive } \\
\text { Characters as Abbreviation } \\
\text { (NCA) }\end{array}$} & & & map & $\mathrm{mp}$ \\
\hline & & & button & $\mathrm{bt}$ \\
\hline & & & load & ld \\
\hline & & & list & 1s \\
\hline \multirow[t]{2}{*}{ Abbreviation Type } & $\mathbf{n}=\mathbf{3}$ & $n=4$ & $\mathbf{n}=\mathbf{3}$ & $n=4$ \\
\hline & Name & Abbr. & Name & Abbr. \\
\hline \multirow{4}{*}{$\begin{array}{l}\text { n-Consecutive Characters } \\
\text { as Abbreviation (CCA) }\end{array}$} & string & str & string & str \\
\hline & buffer & buf & buffer & buf \\
\hline & object & obj & object & obj \\
\hline & array & arr & array & arr \\
\hline \multirow{4}{*}{$\begin{array}{c}\text { n-Nonconsecutive } \\
\text { Characters as Abbreviation } \\
\text { (NCA) }\end{array}$} & source & src & source & src \\
\hline & event & evt & event & evt \\
\hline & message & $\mathrm{msg}$ & message & $\mathrm{msg}$ \\
\hline & button & bth & button & btn \\
\hline
\end{tabular}

\section{Algorithms for Extracting Abbreviations}

Once we have discovered these abbreviations patterns, we are interested in developing algorithms to extract all abbreviations along with their intended words from GitHub. Because nearly $60 \%$ of unigrams are used for naming variables (see Table 2), we would like to extract abbreviations for naming variables. The steps to generate such an abbreviation list are described as follows:

1. Extract all pairs (variable name, the type of the variable name) from GitHub. They are candidates of pairs for extracting an English word and its abbreviation. The basic assumption to compute abbreviations is: when naming a variable, developers more likely to choose an abbreviation based on the type of the variable. The type can be a class, an interface, a primitive, or an array. For example, we may use the abbreviations n, $i t$, and $i$, to represent the instance of Node class, Iterator interface, and integer primitive, respectively.

2. Segment the variable name and variable name type in each pair. The step produces two unigram sets. Each set contains one or more unigrams separated from the variable name and the variable names type, respectively.

3. Find the abbreviations. Pick $w$ and $a$ from the two unigram sets, respectively, and then count the frequency of the pair $(w, a)$ if isAbbre $(w, a)$ returns true. Note that, based on our experience, if $\mathrm{a}$ is an abbreviation of $\mathrm{w}, \mathrm{a}$ is a set. For example, the letter $\mathrm{n}$ can be the abbreviation of classes Node and Number. On the other hand, a type can be represented by multiple abbreviations. For example, the class Node can be represented by $\mathrm{n}$ and $\mathrm{nd}$. It depends on individual developer's preference. The function isAbbre checks the patterns one and four, and then calls a function isconsistent, which implements the first three patterns recursively.

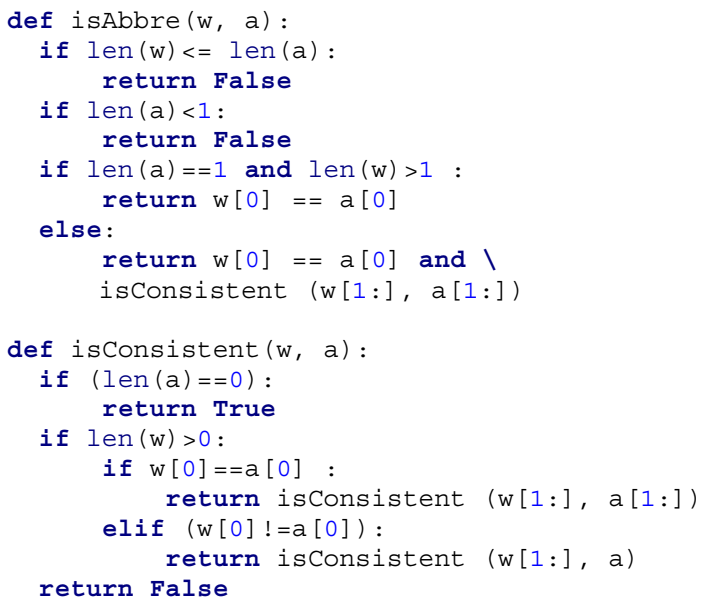

\section{Top Abbreviation Names}

We have extracted 62 million abbreviations from 419 million variable names. It indicates nearly $15 \%$ of variable names use abbreviations. Table 7 shows the top 100 most frequently used abbreviations for naming variables. The results are strongly consistent with developers' naming behaviors. For example, as a developer, we often use $i$ and $e$ for representing int and exception, respectively. Specifically, we have made the following conclusions based on the extracted abbreviations from the GitHub repository: 
- Only five abbreviations have length greater than four characters among the top 100 most frequently used abbreviations for naming variables. The conclusion is consistent with our observation patterns four.

- Abbreviations are widely used for representing the compressed information. Nearly $15 \%$ of variable names use abbreviations.

- Some variable names, such as ioexception, stringbuffer, and bytebuffer are considered as unigrams in [16] because they have relatively high frequencies in natural language corpus and contain no space. For example, ioexception is ranked the 35661 th with a frequency of 595675 in [16].
- Developers may choose different abbreviations for a given word. For instance, for the most frequently used unigram int, developers may pick $i$ or in. However, developers are more likely to use $i$ as abbreviation because the pair (int, i) has a higher frequency than (int, in).

- The same abbreviation can be used for representing different words, e.g., in is used for presenting either int or input. For a given abbreviation in, it is more likely to represent input if we consider the frequency as the only criteria to determine abbreviations.

Table 7. The top 100 most frequently used abbreviations for naming variables

\begin{tabular}{|c|c|c|c|c|c|c|c|c|c|c|c|}
\hline Abb. & Word & $\begin{array}{l}\text { Freq. } \\
\text { (k) }\end{array}$ & Abb. & Word & $\begin{array}{l}\text { Freq. } \\
\text { (k) }\end{array}$ & Abb. & Word & $\begin{array}{l}\text { Freq. } \\
\text { (k) }\end{array}$ & Abb. & Word. & $\begin{array}{c}\text { Freq. } \\
\text { (k) }\end{array}$ \\
\hline $\mathrm{i}$ & int & 9878 & $\mathrm{f}$ & float & 341 & $\mathrm{~m}$ & map & 129 & rect & rectangle & 91 \\
\hline $\mathrm{e}$ & exception & 5584 & ctx & context & 341 & $\mathrm{e}$ & element & 127 & it & int & 87 \\
\hline $\mathrm{s}$ & string & 1575 & conn & connection & 322 & cal & calendar & 123 & caps & capabilities & 87 \\
\hline str & string & 1069 & rs & result & 308 & $\mathrm{~m}$ & method & 120 & $\mathrm{mgr}$ & manager & 86 \\
\hline 1 & long & 1061 & $\mathrm{f}$ & file & 297 & 1 & list & 120 & $\mathrm{f}$ & field & 85 \\
\hline obj & object & 1037 & $\mathrm{msg}$ & message & 294 & re & recognition & 119 & $\mathrm{cmd}$ & command & 85 \\
\hline ex & exception & 937 & $\mathrm{~b}$ & boolean & 273 & $q$ & query & 111 & $\mathrm{cls}$ & class & 81 \\
\hline 0 & object & 781 & ioe & ioexception & 263 & $\mathrm{c}$ & collection & 108 & img & image & 80 \\
\hline $\mathrm{e}$ & event & 561 & $\mathrm{sb}$ & stringbuffer & 253 & $\mathrm{e}$ & encoding & 108 & $\mathrm{~s}$ & session & 79 \\
\hline it & iterator & 535 & $\mathrm{i}$ & iterator & 245 & $\mathrm{t}$ & thread & 106 & params & parameters & 78 \\
\hline in & input & 522 & $\mathrm{n}$ & node & 233 & btn & button & 104 & br & bufferedreader & 77 \\
\hline $\mathrm{V}$ & view & 522 & $\mathrm{db}$ & database & 233 & elem & element & 104 & comp & component & 77 \\
\hline out & output & 492 & attrs & attributeset & 217 & $\mathrm{c}$ & cursor & 103 & $\mathrm{~h}$ & handler & 77 \\
\hline evt & event & 487 & props & properties & 210 & 1 & listener & 103 & $\mathrm{e}$ & entity & 77 \\
\hline in & int & 470 & $\mathrm{fs}$ & filesystem & 205 & $\mathrm{~m}$ & manager & 102 & $\mathrm{~m}$ & message & 76 \\
\hline $\log$ & logger & 437 & prot & protocol & 199 & con & connection & 101 & env & environment & 76 \\
\hline doc & document & 436 & $\mathrm{p}$ & point & 193 & attr & attribute & 100 & $\mathrm{~m}$ & matcher & 76 \\
\hline conf & configuration & 423 & config & configuration & 191 & app & application & 99 & loc & location & 75 \\
\hline $\mathrm{g}$ & graphics & 420 & $\mathrm{ch}$ & char & 176 & prefs & preferences & 97 & cert & certificate & 74 \\
\hline $\mathrm{b}$ & byte & 414 & $\mathrm{e}$ & entry & 171 & buffer & bytebuffer & 96 & $\mathrm{v}$ & visitor & 74 \\
\hline $\mathrm{t}$ & throwable & 401 & stmt & statement & 153 & $\mathrm{t}$ & type & 92 & $\mathrm{c}$ & context & 73 \\
\hline $\mathrm{c}$ & char & 399 & req & request & 152 & $\mathrm{c}$ & component & 92 & $\mathrm{p}$ & player & 73 \\
\hline $\mathrm{sb}$ & stringbuilder & 377 & $\mathrm{c}$ & class & 150 & $\mathrm{e}$ & enumeration & 92 & addr & address & 73 \\
\hline $\mathrm{d}$ & double & 372 & $\mathrm{~V}$ & vector & 144 & $\mathrm{e}$ & error & 91 & $\mathrm{a}$ & array & 72 \\
\hline iter & iterator & 346 & ref & reference & 131 & sql & sqlexception & 91 & nfe & numberformatexception & 71 \\
\hline
\end{tabular}

\section{E. Statistical Properties of Abbreviations}

When developers decide to use an abbreviation to represent its original intended word, they need to make two decisions: (1) determine which type of abbreviation they want to choose, either CCA or NCA (2) determine how much effort they want to save if abbreviations are used compared to original intended English words. Two statistical properties of abbreviations need to be studied to understand the decision process:

- The percentage of CCA versus NCA is used to help us understand how likely developers are to choose CCA or NCA. The percentage of CCA and NCA is the probability of developers to choose CCA and NCA for a given word w. Formally, we compute $P(E)$, where $E$ is an event of choosing an abbreviation for $\mathrm{w}$.

- $\quad$ The Typing Effort Saving (TES). TES computes how much effort developers can save if abbreviations are used. Formally, the TES value for a given pair ( $a$, w) is defined as:

$$
\operatorname{TES}(a, w)=1-\frac{\operatorname{lenth}(a)}{\operatorname{length}(w)}
$$

For example, TES ( $i$, int $)=66.67 \%$, and TES (in, int) $=33.33 \%$. The range of the TES is between 0 and 1 , excluding 0 and 1 . Zero means no abbreviation is used.

Table 8 shows the total number of $62253 \mathrm{k}$ abbreviations used for naming variable names. Nearly $84 \%$ of the abbreviations are CCA, i.e., $\mathrm{P}(\mathrm{E}=\mathrm{CCA})=84 \%$. The number of unique abbreviations is $103 \mathrm{k}$. Over $62 \%$ of these unique abbreviations are CCA. Figure 2 shows the frequency distribution of TES. The x-axis represents the ratio of TES. The $y$-axis is the frequency of TES measured in percentage. For example, let $X$ be the event of computing TES $(\mathrm{a}, \mathrm{w})$, then the frequency of $\operatorname{TES}(i, i n t)$ is 
$\mathrm{P}(\mathrm{X}=\mathrm{TES}(\mathrm{i}$, int $))=\mathrm{P}(\mathrm{X}=66.67 \%)=19 \%$. Overall, this figure indicates that $75 \%$ of the abbreviations have a TES between $50 \%$ and $89 \%$. It implies that developers often use no more than the half size of the English word as abbreviations.

Table 8. The total number abbreviations extracted from variables

\begin{tabular}{|c|c|c|c|c|}
\hline Abbreviation Type & \multicolumn{2}{|c|}{ Unique Abb. (k) } & \multicolumn{2}{c|}{ Total Abb. (k) } \\
\cline { 2 - 5 } & $\#$ & $\mathbf{\%}$ & $\#$ & $\mathbf{\%}$ \\
\hline $\begin{array}{c}\text { n-Consecutive Characters } \\
\text { Abbreviation (CCA) }\end{array}$ & 64 & 62.17 & 52234 & 83.91 \\
\hline $\begin{array}{c}\text { n-Nonconsecutive Characters } \\
\text { Abbreviation (NCA) }\end{array}$ & 39 & 37.83 & 10019 & 16.09 \\
\hline Total & 103 & & 62253 & \\
\hline
\end{tabular}

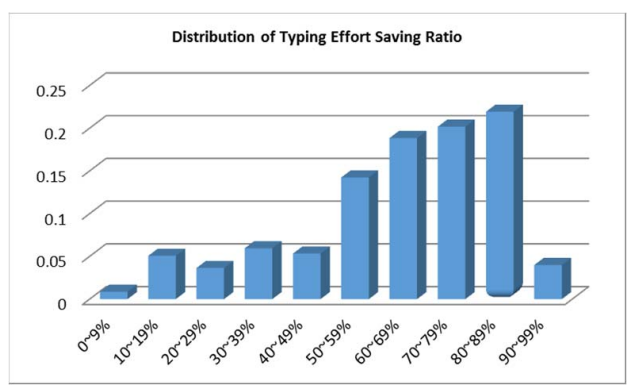

Figure 2. Distribution of TES

It is worth noting that one can argue that abbreviation convention [18] [19]is another reason to naming abbreviation, e.g., the string re should be used as the abbreviation of the word result due to the convention. However, there are several reason not to consider convention during programming:

(1) Unless there is a wide-accepted list of standard abbreviations in source code, such as int and integer, it is challenge for developers to think of abbreviation convention during development. For the re example, other people may use the string res as the abbreviation for result, as the string res has more readability than re. Others may also argue the string res can only be used for representing word response.

(2) The four patterns have already formed the foundation for abbreviation convention.

(3) The abbreviation ranking in Table 7 can be used as a reference for a standard abbreviation convention.

\section{F. Size Distribution of Multigram Names}

Besides unigrams, developers often use multigrams to name identifiers (constructs) to improve the readability of their code. The size of a multigram is the number of the unigrams that the multigram consists of. The size distribution of multigrams indicates how likely developers name constructs with various sizes.

To compute the size of a multigram, we first utilize a word segmentation algorithm to break the multigram into segments, and then we simply count the number of the segments. Figure 3 shows the size distribution of variable names, method names, and class names. The $\mathrm{x}$-axis is the size of a name. The $\mathrm{y}$-axis is the frequency of the name size in percentage. The size distribution is generated using the 701 million names that are shown in Table 1. The figure shows:

- The most likely choice, i.e., with the probability of $65 \%$, developers use a unigram to name a variable.

- $34 \%$ of variable names are multigrams.

- The majority, i.e., $69 \%$, of multigram variable names has the size of two.

- When naming methods and classes, developers often use, i.e., with the probability of over $80 \%$, multigrams.

- There is no significant difference regarding size when naming methods and classes.

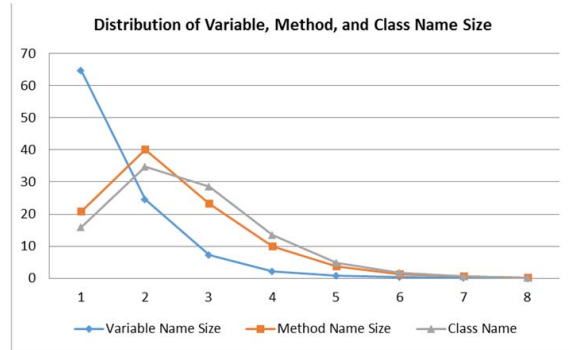

Figure 3. Size distribution of variable, method, and class names

\section{EMPIRICAL STUDY: ABBREVIATION EXPANSION USING UNIGRAMS}

Although the use of abbreviations can help developers to implement faster, it may create confusion in the source code and therefore a decrease in program readability. For example, the variable abbreviation $\mathrm{v}$ in Table 7 can be interpreted as view $(522 \mathrm{k})$, vector $(144 \mathrm{k})$, or visitor $(74 \mathrm{k})$. Different approaches [20] have been proposed to expand abbreviations, and however, these approaches have not considered the properties of program languages in the context of linguistics. In this empirical study, we demonstrate that utilizing the properties of unigrams collected from the ultra-large scale source code repository can solve domain specific problem, such as expanding the abbreviations used in a source code.

Table 9. Example candidate words for abbreviation $r e$ and their frequencies

\begin{tabular}{|c|c|c|c|c|}
\hline \multirow[t]{3}{*}{ Candidate } & \multicolumn{4}{|c|}{ Frequency in unigram } \\
\hline & \multicolumn{2}{|c|}{ SC } & \multicolumn{2}{|c|}{ NC } \\
\hline & $\#$ & $\%$ & $\#$ & $\%$ \\
\hline result & $3,983,282$ & 0.626 & $127,425,045$ & 0.022 \\
\hline request & $1,758,393$ & 0.277 & $124,620,318$ & 0.021 \\
\hline response & $1,333,290$ & 0.210 & $84,065,293$ & 0.014 \\
\hline resource & 822,922 & 0.129 & $99,964,083$ & 0.017 \\
\hline read & 443,324 & 0.070 & $322,331,766$ & 0.055 \\
\hline repository & 211,294 & 0.033 & $8,892,664$ & 0.002 \\
\hline rule & 531,175 & 0.084 & $97,658,641$ & 0.017 \\
\hline range & 374,764 & 0.059 & $128,314,924$ & 0.022 \\
\hline
\end{tabular}

\section{A. A Probabilistic Language Model to Expand Abbreviations}

Assume that we have a task that needs to figure out what the abbreviation re stands for. Based on the patterns of English words and their abbreviations, Table 9 lists eight examples of possible words that match the abbreviation 
computed from the source code and natural language corpus [16]. These words are referred to as the candidates of the abbreviation. Table 9 also includes the candidates' corresponding frequencies in two sets of unigrams, respectively. Note that the total number of unigrams in the source code and natural language corpus are 636 million and 588,118 million, respectively.

If only considering the frequency, we choose the English words result and read as the intended words in the domain of programming and the natural languages, respectively. They have the highest probability regarding frequency. However, these results lack compelling evidence. We use the Naive Bayes probabilistic model instead to solve the uncertain problem. Formally, the problem can be described as follows: given an abbreviation, a, determine what word $c$ was the most likely original word. For example, if a is re, then request is $\mathrm{C}$, which is the most likely word in the domain of programming languages. The language model for choosing the best candidate among all candidates is shown as follows:

$\operatorname{argmax}_{c} P(c \mid a)=\operatorname{argmx}_{c} P(a \mid c) * P(c)$

where $P(c)$ is the probability of $c$, the candidate English word in the source code unigrams.

\section{$\operatorname{argmax}_{c} P(c \mid a)$ : the highest $P(c \mid a)$.}

$\mathrm{P}(\mathrm{a} \mid \mathrm{C})$ is the probability that a developer will use the abbreviation $a$ to represent $c$. It is called the abbreviation representation model. The representation model is based on the frequency distribution of TES ratio that is shown in Figure 2, and the distribution of abbreviation types (which we will discuss in details in subsection $\mathrm{C}$ ). Note that experts may disagree with the abbreviation representation model. Therefore there is no complete model. We only use the model to demonstrate the importance of the unigrams that are generated from source code. In other words, the properties of unigrams can help us to solve domain specific problems.

\section{B. Search Candidates for Language Model}

It is unwise to pick up all English words in the unigrams that match the abbreviation patterns as candidates because there are too many possible matches. For example, the candidates for the given abbreviation re will include all the English words that start with $r$ and contain e (consecutively or nonconsecutively). In fact, it makes more senses to pick up candidates based on the locations of the given abbreviation in the source code. There are two approaches to search candidates: static and dynamic approaches. Static approach searches for candidates within a certain radius of the abbreviation, e.g., the method or class in which the abbreviation is used. Dynamic approach generates the data flow diagrams or control flow diagrams from source code first and then searches for the candidates within the radius of the abbreviations in these diagrams. Once the candidates search radius is determined for the given abbreviation a, we pick each English word $c$ in the radius, using isAbbre $(c, a)$ to test if the $\mathrm{C}$ is candidate. For example, assume we have chosen the static approach where the method is the candidate search radius for the given abbreviation re. Table 10 contains ten candidate unigrams for the abbreviation re in a method. The first eight unigrams are CCA and the last two are NCA.

\section{Compute the Best Candidates as the Expanded Word}

Our goal is to compute $\operatorname{argmax}_{\mathrm{C}} \mathrm{P}(\mathrm{c} \mid \mathrm{a})$. The challenge of computing $\operatorname{argmax}_{C} \mathrm{P}(\mathrm{c} \mid \mathrm{a})$ is to compute $\mathrm{P}(\mathrm{a} \mid \mathrm{C})$ basedon formula 2 . We could simply use the frequency of pair $(a, c)$ to compute $P(a \mid c)$. However, the pair $(a, c)$ may not exist. Hence we use the two statistical properties of abbreviation that were discussed earlier to generalize the process of calculating $P(a \mid c)$. The properties describe how developers choose an abbreviation for a given English word. Formally,

$$
P(a \mid c)=P(E=\text { AbbType }) \times P(X=T E S(a, c))
$$

Table 10. Computing the best candidate for expanding abbreviation $r e$ using different unigrams generated from source code and natural language corpus

\begin{tabular}{|c|c|c|c|c|c|c|c|c|c|c|c|}
\hline \multirow{2}{*}{$\begin{array}{l}\text { Abbr. } \\
\text { (a) }\end{array}$} & \multirow{2}{*}{$\begin{array}{l}\text { Candidate } \\
\text { unigram } \\
\text { (c) }\end{array}$} & \multirow[t]{2}{*}{\begin{tabular}{l|l|l}
$\mathbf{a}$ & $\mathbf{c}$
\end{tabular}} & \multirow{2}{*}{$\begin{array}{l}\text { Abb. } \\
\text { Type }\end{array}$} & \multirow[t]{2}{*}{ P(AbbType) } & \multirow{2}{*}{$\begin{array}{c}\text { TES } \\
(a, c)\end{array}$} & \multirow{2}{*}{$\begin{array}{c}\mathbf{P} \\
(\operatorname{TES}(\mathbf{a}, \mathbf{c}))\end{array}$} & \multirow{2}{*}{$\underset{(a \mid c)}{p}$} & \multicolumn{2}{|c|}{$\mathbf{p}(\mathbf{c})$} & \multicolumn{2}{|c|}{$\mathbf{P}(\mathbf{c} \mid \mathbf{a})=\mathbf{P}(\mathbf{a} \mid \mathbf{c}) * \mathbf{P}(\mathbf{c})$} \\
\hline & & & & & & & & $\mathrm{SC}$ & $\mathrm{NC}$ & SC & $\mathrm{NC}$ \\
\hline re & result & re $\mid$ result & CCA & 0.84 & 0.67 & 0.19 & 0.1596 & 0.6263 & 0.0217 & 0.09995748 & 0.00346332 \\
\hline re & request & \begin{tabular}{l|l} 
re & request \\
\end{tabular} & $\mathrm{CCA}$ & 0.84 & 0.71 & 0.2 & 0.168 & 0.2765 & 0.0212 & 0.046452 & 0.0035616 \\
\hline re & response & \begin{tabular}{l|l} 
re & response
\end{tabular} & $\mathrm{CCA}$ & 0.84 & 0.75 & 0.2 & 0.168 & 0.2097 & 0.0143 & 0.0352296 & 0.0024024 \\
\hline re & resource & \begin{tabular}{l|l} 
re & resource
\end{tabular} & CCA & 0.84 & 0.75 & 0.2 & 0.168 & 0.1294 & 0.017 & 0.0217392 & 0.0028560 \\
\hline re & reader & re $\mid$ reader & $\mathrm{CCA}$ & 0.84 & 0.67 & 0.19 & 0.1596 & 0.0957 & 0.0087 & 0.01527372 & 0.00138852 \\
\hline re & read & re $\mid$ read & $\mathrm{CCA}$ & 0.84 & 0.5 & 0.14 & 0.1176 & 0.0697 & 0.0548 & 0.00819672 & 0.00644448 \\
\hline re & results & \begin{tabular}{l|l|} 
re & results \\
\end{tabular} & CCA & 0.84 & 0.71 & 0.2 & 0.168 & 0.0624 & 0.0046 & 0.0104832 & 0.0007728 \\
\hline re & repository & \begin{tabular}{l|l} 
re & repository
\end{tabular} & $\mathrm{CCA}$ & 0.84 & 0.8 & 0.22 & 0.1848 & 0.0332 & 0.0015 & 0.00613536 & 0.0002772 \\
\hline re & rule & re $\mid$ rule & NCA & 0.16 & 0.5 & 0.14 & 0.0224 & 0.0835 & 0.0167 & 0.0018704 & 0.00037408 \\
\hline re & range & re $\mid$ range & $\mathrm{NCA}$ & 0.16 & 0.67 & 0.19 & 0.0304 & 0.0589 & 0.0218 & 0.00179056 & 0.00066272 \\
\hline
\end{tabular}

Bold Numbers: Top candidates Italic Numbers: Different ranks between P (c) and P (c|a)

Table 10 shows the expanding of abbreviations using different unigrams. Our observations are as follows:

- The best candidates for the given abbreviation re may be different in different language domains. The words results and read have the highest probabilities of being the original English words in source code and natural language corpus, respectively. Bold numbers are the highest probabilities of candidates in source and natural language corpus, respectively.

- Regardless of the type of language, the frequency of a unigram is an essential fact in determining the best candidate. For example, both of the best candidates for re have the highest frequencies among all candidates in their corresponding unigram models. 
- TES determines the best candidate if two of the same type of candidates have similar frequency in its language domains. For example, the two words read and results in the programming language domain are both CCA, the word results is considered as the better candidate even the word read has a slightly higher frequency than results does. Similarly, the word request is a better candidate than result in the domain of natural language (see Italic numbers in Table 9).

Note that (1) we only consider expanding a given abbreviation that is chosen from a simple English word due to the page limitation. The comprehensive approach to expanding other types of abbreviations, including abbreviations selected from multigram names, as well as the empirical study will be addressed in the future work and (2) we only demonstrate the importance of utilizing the properties of unigrams to solve well-known problems, such as understanding abbreviations. Empirical study regarding the comparison of different approaches for expanding abbreviations will be included in the future work.

\section{CONCLUSION}

Unigrams collected from source code repository are useful for dealing software development problems, such as understanding the behaviors of developers and expanding abbreviations to improve the code readability. We have extracted 0.70 billion names from nearly 0.7 million projects. The names include variable, method, and class names. A total of 1.01 billion unigrams are generated from these constructs. In addition, 62 million abbreviations are extracted from 419 million variable names. To demonstrate the importance of unigrams, we have analyzed the patterns of how developers choose abbreviations, and then, we have used the properties of unigrams to expand abbreviations to original English words. The completed analysis results, including unigrams collected from variable, method, and class names, as well as abbreviations, can be accessed at https://goo.gl/HUz06W. The raw data extracted from GitHub can be accessed at https://goo.gl/nxzqHd. All variable names, method names, and class names are stored under the folders named variables, methodName, and className. Concerning the future work, we plan to expand our approach to collect and analyze bigrams and trigrams from source code repository. We also plan to conduct a larger empirical study to expand various types of abbreviations.

\section{REFERENCES}

[1] D. Roth, "Learning to Resolve Natural Language Ambiguities: A Unified Approach," in Fifteenth National Conference on Artificial Intelligence, Madison, Wisconsin, 1998.

[2] H. Kamp and U. Reyle, From Discourse to Logic: Introduction to Modeltheoretic Semantics of Natural Language, Formal Logic and Discourse Representation Theory, Springer Science Business Media, 2013.

[3] N. Chomsky, "On certain formal properties of grammars," Information and Control, vol. 2, no. 2, p. 137-167, 1959.
[4] M. L. Scott, Programming Language Pragmatics, 3rd ed. ed., San Francisco, CA, USA: Morgan Kaufmann Publishers Inc., 2009.

[5] "GitHub," [Online]. Available: www.github.com. [Accessed 20 5 2016].

[6] S. Yoo, "Boa Language," [Online]. Available: http://web.cs.ucla.edu/ shyoo1st/boa/. [Accessed 28 2016].

[7] "Linguistics Data Consortium," Google.com, 2006. [Online]. Available: https://catalog.ldc.upenn.edu/LDC2006T13. [Accessed 26 201].

[8] "Google Ngram Viewer - Google Books," Google.com, 52012. [Online]. Available: https://books.google.com/ngrams. [Accessed 205 2016].

[9] M. Allamanis and C. Sutton, "Mining source code repositories at massive scale using language modeling," in 10th IEEE Working Conference on Mining Software Repositories (MSR '13), 2013.

[10] M. Allamanis, E. T. Barr, C. Bird and C. Sutton, "Learning natural coding conventions," in Proceedings of the 22nd ACM SIGSOFT International Symposium on Foundations of Software Engineering (FSE 2014), 2014.

[11] M. Allamanis, E. T. Barr, C. Bird and C. Sutton, "Suggesting accurate method and class names," in In Proceedings of the 2015 10th Joint Meeting on Foundations of Software Engineering (ESEC/FSE 2015), 2015.

[12] V. Raychev, M. Vechev and A. Krause, "Predicting Program Properties from "Big Code"," in roceedings of the 42nd Annual ACM SIGPLAN-SIGACT Symposium on Principles of Programming Languages, 2015.

[13] R. Dyer, H. . A. Nguyen, H. Rajan and T. N. Nguyen, "Boa: A Language and Infrastructure for Analyzing Ultra-Large-Scale Software Repositories," in the 35th International Conference on Software Engineering, Francisco, CA, 2013.

[14] T. Segaran and J. Hammerbacher, Beautiful Data: The Stories Behind Elegant Data Solutions, O'Reilly Media, 2009.

[15] "Hadoop," The Apache Software Foundation, [Online]. Available: http://hadoop.apache.org/. [Accessed 205 2016].

[16] G. Jenks, "wordsegment 0.6.2," [Online]. Available: https://pypi.python.org/pypi/wordsegment. [Accessed $22 \quad 5$ 2016].

[17] R. P. Buse and W. Weimer, "Learning a Metric for Code Readability," IEEE Transactions on Software Engineering, vol. 36, no. 4, pp. 546-558, 2010.

[18] D. Pierret and D. Poshyvanyk, "An empirical exploration of regularities in open-source software lexicons," in The International Conference on Program Comprehension, Vancouver, Canada, 2009.

[19] M. White, C. Vendome, M. L. Vásquez and D. Poshyvanyk, "Toward Deep Learning Software Repositories," in International Conference on Mining Software Repositories , Florence, Italy, 2015.

[20] E. Hill, Z. P. Fry, H. Boyd, G. Sridhara, Y. Novikova, L. Pollock and K. Vijay-Shanker, "AMAP: Automatically Mining Abbreviation Expansions in Programs to Enhance Software Maintenance Tools," in the 2008 international working conference on Mining software repositories, Leipzig, Germany, 2008. 